\title{
The effectiveness of a Botulinum Toxin A infiltration in the management of bicipital cramps after arthroscopic biceps tenotomy
}

\author{
Marieke Torrekens, Bert VAnMIerlo, Tom VAN IsACKer
}

From the Department of Orthopaedic Surgery, St Lucas and St Jan General Hospital, Bruges, Belgium

\begin{abstract}
A challenging complication of arthroscopic biceps surgery is the persistent painful cramping of the biceps. There is a paucity of data upon nonsurgical treatment of this debilitating complication. We propose an intramuscular injection of botulinum toxin A (BTX-A) for painful bicipital cramping after tenotomy of the long head of the biceps brachii tendon (LHBT). Ten patients with a painful Popeye sign after tenotomy of LHBT, were treated with intramuscular injection of 100 IU of BTX-A. Mean patient age was 56 years and mean time from surgery to infiltration was 317 days. The Quick Disabilities of the Arm, Shoulder and Hand (QuickDASH) score was obtained. Pain was objectified by a visual analogue scale (VAS). Patient satisfaction was described as excellent, good, satisfactory, or poor, three and six months after injection. Mean VAS prior to infiltration was 6.8 and decreased significantly to 2.6 at follow-up. Mean QuickDash was $\mathbf{5 4 . 0 4}$ prior to infiltration and decreased to $\mathbf{1 9 . 8 4}$ at follow-up. Patient satisfaction was excellent in 9 and good in 1 patient. We report a significant pain reduction and functional improvement following BTX-A infiltration as treatment of painful bicipital cramping after tenotomy of LHBT.
\end{abstract}

Keywords: bicipital cramping; LHBT; BTX-A; botox; tenotomy; shoulder arthroscopy; rotator cuff.

Declaration of conflicting interests: None of the authors have any conflicting interests concerning the topic of this study. Funding: This work was not supported by any funding. Ethical approval and informed consent details: Ethical approval was obtained from the ethical committee of $A Z$ Sint-Lucas, Brugge (EudraCT-number : 2019-000498-22).

\section{INTRODUCTION}

Lesions of the tendon of the long head of the biceps brachii (LHB) have been the subject of intense research for decades. They comprise a spectrum of pathologies, originating from inflammatory tenosynovitis to degenerative tendinopathy caused by overuse, trauma, or instability (1). Pain in the anterior part of the shoulder, at rest as well as on rotation of the shoulder, radiating pain down the arm, and paresthesia are frequent symptoms. Varying degrees of disability are observed $(2,3,4)$. Treatment options are initially conservative, including nonsteroidal anti-inflammatory drugs, adjustment of activities, rest, physical therapy, and corticosteroid injections (4). If conservative management is unsuccessful, a range of surgical techniques has been suggested. Although the most appropriate management has

Marieke Torrekens ${ }^{1}$,

- Bert Vanmierlo²,

- Tom Van Isacker ${ }^{3}$

${ }^{1}$ Department of Orthopaedic Surgery, AZ Jan portaels, Gendarmeriestraat 65, Vilvoorde, Belgium.

${ }^{2}$ Department of Orthopaedic Surgery, AZ Delta, Deltalaan 1, Roeselare, Belgium.

${ }^{3}$ Department of Orthopaedic Surgery, AZ Sint-Lucas, SintLucaslaan 29, Brugge, Belgium.

Correspondence : Marieke Torrekens, Department of Orthopaedic Surgery, AZ Jan portaels, Gendarmeriestraat 65, Vilvoorde, Belgium. Phone: +32 472825507.

Email : marieke.torrekens@azjanportaels.be

- 2021, Acta Orthopædica Belgica.

Acta Orthopædica Belgica, Vol. 87 - 4 - 2021 
been the subject of debate, arthroscopic removal of the diseased intra-articular portion of the LHB, by tenotomy or tenodesis, is increasingly accepted (3). Both techniques have similar outcomes (47). Despite the good functional results, reported complications of biceps surgery are the Popeye sign and loss of strength in elbow flexion and forearm supination (3-9). The Popeye deformity occurs due to a distal displacement of the long head of the biceps after spontaneous rupture or tenotomy, and the biceps muscle crops to a round ball as seen in the illustration (Fig. 1). Referring to the cartoon personage, this deformity is called a Popeye arm or Popeye sign. Most of the time, this deformity is pain free, but can cause extremely painful cramps in the muscle belly. The painful Popeye sign, which is more frequent in arthroscopic biceps tenotomy, significantly reduces the quality of life, and symptoms tend to be persistent (10-12).

Some authors suggest a surgical conversion to subpectoral tenodesis of the LHB to treat chronic bicipital pain and contracture after biceps tenotomy $(13,14)$.

There is a paucity of data on outcomes of nonsurgical treatment measures of this debilitating complication (15).

In this manuscript, we evaluate the clinical effectiveness of intramuscular injection of botulinum toxin A (BTX-A), in the treatment of painful bicipital cramping after tenotomy of the LHB.

\section{MATERIAL AND METHODS}

Between august 2017 and march 2019, ten patients with a painful Popeye sign, which resulted from tenotomy of the LHB tendon, were treated with intramuscular injection of BTX-A. Patient selection was based upon the presence of severe bicipital pain in the muscle belly of the biceps brachii at rest and during activity, that did not respond to conservative treatment and that persisted for more than three months. Patients with other causes of persistent shoulder pain after tenotomy were excluded. Conservative treatment included physical therapy and non-steroidal anti-inflammatory drugs. All patients underwent treatment by injection of $100 \mathrm{IU}$ of BTX-A in the painful muscle belly of

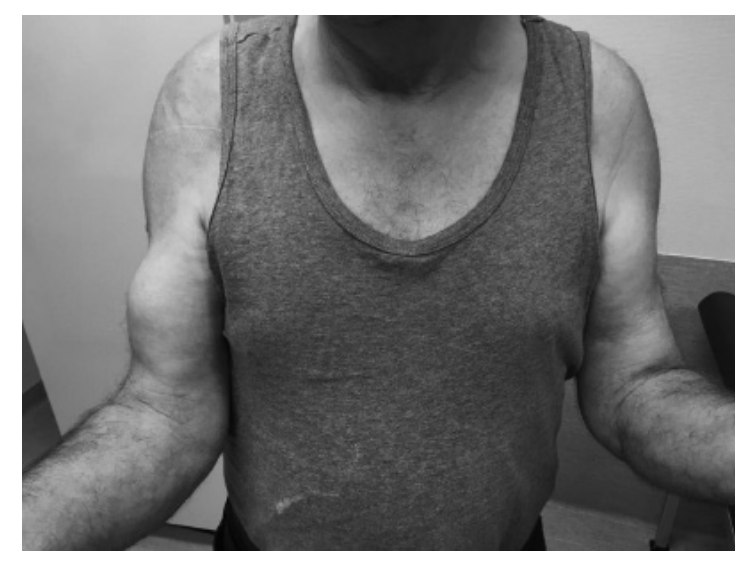

Figure 1. - A patient with a Popeye deformity in the right arm due to a distal displacement of the long head of the biceps after tenotomy.

the biceps brachii. These 100 IU of BTX-A were dissolved in $2.5 \mathrm{cc}$ of a sterile saline $(\mathrm{NaCl} 0.9 \%)$ solution and were distributed over the contracted muscle belly through four separate puncture holes, and by injecting in a multidirectional fashion. Patient assessment occurred prior to injection and at a three months follow-up consultation. The Quick Disabilities of the Arm, Shoulder and Hand (QuickDASH) score was obtained to determine overall function (16). Pain was objectified by a visual analogue scale (VAS): a score of 0 indicated no pain, and a score of ten the worst pain imaginable by the patient (17). Patient satisfaction was described as either excellent, good, satisfactory, or poor, three months after injection. A final clinical evaluation was performed at six months of follow-up.

VAS and QuickDASH scores were compared between baseline and three months after injection, with a paired samples t-test. The significance for all outcomes was defined as $\mathrm{p}<0.05$.

\section{RESULTS}

Ten patients were treated with local intramuscular administration of BTX-A injection, for a symptomatic postoperative Popeye sign. The male to female ratio was one to nine. Mean age was 56 years old (range 41 to 69), and mean time lapse from surgery to infiltration was 317 days (range 151 to 700 ). Mean VAS prior to infiltration was 6.8 $+/-1.2$ and this decreased to $2.7+/-1.9$ at the three 
months follow-up consultation. This difference was statistically significant $(\mathrm{p}<0.0001)$. Mean QuickDash was $54.05+/-12.0$ prior to infiltration and decreased to $19.09+/-9.1$ at follow-up. Once again significance was obtained $(p<0.0001)$. No complications were reported in this study. Nevertheless, all patients mentioned minor and transient diminution of force when exerting elbow flexion and forearm supination. Patient satisfaction was excellent in nine and good in one patient. Pain reduction started two weeks after administration of BTX-A and lasted for four to six months. At a six months follow-up consultation, seven out of ten patients preferred a second intramuscular administration of BTX-A above referral for surgical treatment, when discussing the therapeutic options. The remaining three patients were comfortable and did not seek any further treatment.

\section{DISCUSSION}

The intramuscular administration of BTX-A injection for a symptomatic postoperative Popeye sign, caused a significant improvement in terms of VAS and QuickDash score. BTX-A is widely used for treating orthopaedic disorders that involve muscular spasm, as

it reduces muscle activity by inhibiting the release of acetylcholine at the neuromuscular junction level (11,18-20). Since 1989, BTX-A infiltration has been approved for therapeutic and esthetical treatment purposes (21). Although it is known as a toxic biologic agent (21), the administration of small doses for medical use, is considered safe (22-24).

BTX -A has a proven efficiency in the treatment of a variety of neurological and non- neurological disorders (22-24), such as blepharospasm (25), cervical dystonia (25), headache (26), tremors (27), spasticity (post-stroke spasticity, spasticity in Cerebral palsy), and even plantar fasciitis (28). Most orthopaedic disorders that benefit from the administration of BTX-A, involve spasticity and pain (11,18-20), which is no different in our patient series. Following arthroscopic biceps tenotomy, the majority of the patients are pain free (2-10). However, a minor group of patients develop persistent and painful cramping of the biceps muscle that is resistant to conventional conservative management. The incidence of a symptomatic Popeye sign after arthroscopic biceps tenotomy ranges from three to $38 \%(5,7,8,10)$. These muscular spasms have a negative impact on the quality of life (12).

Our case series demonstrates the value of BTX-A in the conservative management of bicipital pain and contracture after biceps tenotomy. The administration is simple, straightforward, and little invasive. No activity restriction or specific rehabilitation protocol is required following the treatment. None of the patients developed any drugrelated adverse events (11,18-20).

Pain relief after BTX-A administration lasted for four to six months, which is comparable to the duration of the effect of BTX-A when administered for other indications, such as spastic muscles in cerebral palsy $(19,20)$. Seven out of ten patients in our series preferred BTX-A administration above referral for subpectoral tenodesis, when

experiencing recurrency of the symptoms at a 6 months follow-up consultation. Repeating BTX-A administration is safe, efficient, and might even induce an ever- increasing degree of muscular atrophy at each infiltrative cycle. This reduction in bicipital muscular mass, might explain the lower demand for treatment at the six months follow-up consultation.

Surgical tenotomy of the long head of the biceps brachii elicits a decrease of the strength of elbow flexion and forearm supination from respectively eight to $16 \%$ and from eleven to $21 \% \quad(29,30)$. BTX-A infiltration reduces strength as it causes muscle atrophy (20,31-33). Since an avulsed and contracted muscle belly has little to no effect on the mobility nor on the strength of motion, paralyzing this muscle belly will not affect either of them. The subjective sensation of a minor and transient diminution of force when exerting elbow flexion and supination, might be explained by diffusion of the injected BTX-A to the intact muscle belly of the biceps brachii and even to the brachialis muscle. When reviewing literature upon the surgical treatment options of painful bicipital cramping after tenotomy, revisionary surgery and conversion to subpectoral tenodesis of the avulsed tendon is considered a successful approach. Gregory et 
al. (13) reviewed in 2012 a series of 21 patients (mean age, 46.5 years) who underwent a miniopen subpectoral biceps tenodesis for persistent bicipital pain after biceps tenotomy (one out of 21 patients) or failed tenodesis (20 out of 21 patients). Tenodesis was performed into the bicipital groove with an interference screw. The mean time from the initial biceps intervention to revisionary surgery was 19.6 months. With an average follow-up of 33.4 months, eleven out of 21 patients reported significant pain relief and functional improvement. Six patients were lost to follow-up and only one patient was not satisfied with the result. Anthony et al. (14) reported their results in 2015; eleven patients (mean age, 43.3 years) with symptomatic bicipital cramping after biceps tenotomy (six out of eleven patients) or biceps rupture (five out of eleven patients), underwent a mini-open subpectoral biceps tenodesis with interference screw. Ten patients reported an improvement of cosmetic deformity and pain was resolved in eight patients. One patient was lost to follow-up. These results are consistent with those of other studies on the clinical outcome of revision biceps tenodesis for persistent cramping pain in the biceps $(34,35,36)$. In our study ten patients (mean age of 56 years) were treated with local intramuscular administration of BTX-A injection, for a symptomatic postoperative Popeye sign. Mean time lapse from surgery to infiltration was 317 days. We noted a patient satisfaction that was excellent in nine and good in one patient.

In contrast to BTX-A infiltration, revision biceps tenodesis is an invasive procedure and implies the necessity of a rehabilitation protocol with restriction of resisted elbow flexion for a period of six weeks after surgery (13,14,34-36).

Nevertheless, there are several limitations to our study that are worth mentioning. First, our patient group is limited and there is no control group for comparison. Data were collected in a retrospective fashion. We did not set out to do a prospective randomized controlled trial (RCT), since this would require a larger study effort and cost than available. However, it would be interesting to perform a RCT, comparing the intramuscular administration of BTX-A to an alternative placebo treatment, such as the infiltration of a similar volume of $\mathrm{NaCl}$
$0,9 \%$. Second, our study did not include objective measurements of strength, only patient-reported outcomes of improvement in pain and function, which require an objectification of subjective patients (37). Third, the potential benefit of repeated BTX-A infiltrations for persisting cramping in the biceps muscle still has to be confirmed.

In conclusion, our study demonstrates a significant and promising pain reduction and functional improvement following BTX-A infiltration as treatment of painful bicipital cramping after tenotomy of the long head of the biceps muscle.

\section{REFERENCES}

1. Ahrens PM, Boileau P. The long head of biceps and associated tendinopathy. J Bone Joint Surg Br 2007; 89(8): 1001-1009.

2. Hitchcock HH, Bechtol CO. Painful shoulder: observations on the role of the tendon of the long head of the biceps brachii in its causation. J Bone Joint Surg Am.1948; 30A(2): 263-73.

3. Walch G, Edwards TB, Boulahia A, Nové-Josserand L, Neyton L, SzaboI. Arthroscopic tenotomy of the long head of the biceps in the treatment of rotator cuff tears: clinical and radiographic results of 307 cases. J Shoulder Elbow Surg. 2005; 14(3): 238-46.

4. Nho SJ, Strauss EJ, Lenart BA, Provencher MT, Mazzocca AD, Verma NN, Romeo AA. Long head of the biceps tendinopathy: diagnosis and management. $J \mathrm{Am}$ Acad Orthop Surg. 2010; 18(11): 645-56.

5. Boileau P, Basqué F, Valerio L, Ahrens $P$, Chuinard C, Trojani C. Isolated arthroscopic biceps tenotomy or tenodesis improves symptoms in patients with massive irreparable rotator cuff tears. J Bone Joint Surg Am 2007; 89: 747-57.

6. Frost A, Zafar MS, Maffulli N. Tenotomy versus tenodesis in the management of pathologic lesions of the tendon of the long head of the biceps brachii. Am J Sports Med. 2009; 37(4): 828-33. Epub 2008 Sep 1.

7. Wittstein JR, Queen R, Abbey A, Toth A, Moorman CT. III Isokinetic strength, endurance, and subjective outcomes after biceps tenotomy versus tenodesis: a postoperative study. Am J Sports Med. 2011; 39: 857-65.

8. Duff SJ, Campbell PT. Patient acceptance of long head of biceps brachii tenotomy. J Shoulder Elbow Surg 2012; 21: 61-5.

9. Heng'anGe, QiangZhang, Yeqing Sun, JieLi , LinSun, Biao Cheng. Tenotomy or Tenodesis for the Long Head of Biceps Lesions in Shoulders: A Systematic Review and Meta-Analysis. PLoS One. 2015; 10.

10. Laure Pouliquena, Julien Berhouetb, Marion Istvanc, Hervé Thomazeaua, Mickael Roparsa, Philippe Collin. 
Popeye sign: Frequency and functional impact. Orthop Traumatol Surg Res 2018; 104: 817-822.

11. Domenico A. Restivo, Riccardo Vigneri, et al. Efficacy of Botulinum Toxin A for Treating Cramps in Diabetic Neuropathy. Ann Neurol 2018; 84: 682-690.

12. Rozina Bhimani, Lisa Anderson. Clinical Understanding of Spasticity: Implications for Practice. Rehabil Res Pract. 2014; 2014: 279175.

13. Gregory JM, Harwood DP, Gochanour E, Sherman SL, Romeo AA. Clinical outcomes of revision biceps tenodesis. Int J Shoulder Surg. 2012; 6(2): 45-50.

14. Anthony SG, McCormick F, Gross DJ, Golijanin P, Provencher MT. Biceps tenodesis for long head of the biceps after auto-rupture or failed surgical tenotomy: results in an active population. J Shoulder Elbow Surg 2015; 24(2): e36-e40.

15. Phillips BB, Canale ST, Sisk TD, Stralka SW, Wyatt KP. Ruptures of the proximal biceps tendon in middle-aged patients. Orthop Rev. 1993; 22(3): 349-53.

16. Gummesson C, Ward MM, Atroshi I. The shortened disabilities of the arm, shoulder and hand questionnaire (QuickDASH): validity and reliability based on responses within the full-length DASH. BMC Musculoskelet Disord. 2006; 7: 44.

17. Joyce CR, Zutshi DW, Hrubes V, Mason RM. Comparison of fixed interval and visual analogue scales for rating chronic pain. Eur J Clin Pharmacol. 1975; 8(6): 41520.

18. Keizer SB, Rutten HP, Pilot P, Morre HH, Os JJ, Verburg AD. Botulinum Toxin injection versus surgical treatment for tennis elbow: a randomized pilot study. Clin Orthop Relat Res. 2002; 401: 125-131.

19. Koman LA, Mooney JF, Smith B, Goodman A, Mulvaney T. Management of cerebral palsy with botulinum-A toxin: preliminary investigation. J Pediatr Orthop. 1993; 13(4): 489-495.

20. Rafael Fortuna, Monika Horisberger, Marco Aurélio Vaz, Walter Herzog. Do skeletal muscle properties recover following repeat onabotulinum toxin A injections? J Biomech 2013; 46: 2426-2433.

21. Jankovic, J. Botulinum toxin: State of the art. Mov. Disord. 2017, 32, 1131-1138.

22. Simpson DM, Blitzer A, Brashear A, et al. Assessment: botulinum neurotoxin for the treatment of movement disorders (an evidence-based review): report of the Therapeutics and Technology Assessment Subcommittee of the American Academy of Neurology. Neurology 2008; 70: 1699-1706a.

23. Simpson DM, Gracies JM, Graham HK, et al. Assessment: botulinum neurotoxin for the treatment of spasticity (an evidence-based review): report of the Therapeutics and Technology Assessment Subcommittee of the American Academy of Neurology. Neurology 2008; 70: 1691-1698b.

24. Naumann M, So Y, Argoff CE, et al. Assessment: botulinum neu- rotoxin in the treatment of autonomic disorders and pain (an evidence-based review): report of the
Therapeutics and Technology Assessment Subcommittee of the American Academy of Neurology. Neurology 2008; 70: 1707-1714.

25. Simpson DM, Hallett M, Ashman EJ, et al. Practice guideline update summary: botulinum neurotoxin for the treatment of blepharospasm, cervical dystonia, adult spasticity, and headache: report of the Guideline Development Subcommittee of the American Academy of Neurology. Neurology 2016; 86(19): 1818-1826.

26. Gobel H, Heinze A, Heinze-Kuhn K, Jost WH. Evidencebased medicine: botulinum toxin A in migraine and tensiontype headache. J Neurol. 2001; 248 (Supp 1): 34-38.

27. Lotia M, Jankovic J. Botulinum toxin for the treatment of tremor and tics. Semin Neurol 2016; 36(1): 54-63.

28. Ismael V Díaz-Llopis, Carmen M Rodríguez-Ruíz, Sandra Mulet-Perry, Francisco J Mondéjar-Gómez, Jose M Climent-Barberá Francisco Cholbi-LLobel. Randomized controlled study of the efficacy of the injection of botulinum toxin type A versus corticosteroids in chronic plantar fasciitis: results at one and six months. Clin Rehabil 26(7) 594-606.

29. Sturzenegger M, Beguin D, Grunig B, Jakob RP. Muscular strength after rupture of the long head of the biceps. Arch Orthop Trauma Surg 1986; 105: 18-23.

30. Deutch SR, Gelineck J, Johannsen HV, Sneppen O. Permanent disabilities in the displaced muscle from rupture of the long head tendon of the biceps. Scand J Med Sci Sports. 2005; 15: 159-62.

31. Brin, M.F. Botulinum toxin: chemistry, pharmacology, toxicity, and immu- nology. Muscle \& Nerve 1997; (Suppl. 6), S146-68.

32. Molenaers, G., Van Campenhout, A., Fagard, K., De Cat, J., Desloovere, K. The use of botulinum toxin A in children with cerebral palsy, with a focus on the lower limb. J Child Orthop. 2010; 4, 183-195.

33. Schroeder, A.S., Ertl-Wagner, B., Britsch, S., Schroder, J.M., et al.Muscle biopsy substantiates long-term MRI alterations one year after a single dose of botulinum toxin injected into the lateral gastrocnemius muscle of healthy volunteers. Mov Disord 2009; 24, 1494-1503.

34. Ng CY, Funk L. Symptomatic chronic long head of biceps rupture: Surgical results. Int J Shoulder Surg 2012; 6: 108111.

35. Euler SA, Marilee P. Horan, Michael B. Ellman, Joshua A. Greenspoon, Peter J. Millett. Chronic rupture of the long head of the biceps tendon: comparison of 2- year results following primary versus revision open subpectoral biceps tenodesis. Arch Orthop Trauma Surg. 2016; 136: 657-663.

36. Tucker CJ, Dutta AK. Tenodesis of isolated proximal ruptures of the long head of the biceps brachii. Tech Shoulder Elbow Surg. 2009; 10: 72-75.

37. Lohr KN, Zebrack BJ. Using patient-reported outcomes in clinical practice: challenges and opportunities. Qual Life Res. 2009; 18: 99-107. 